\title{
Les observations de poissons tropicaux et le réchauffement des eaux dans l'Atlantique européen
}

\author{
Jean-Claude QUEIRO $^{\text {a* }}{ }^{\text {, Marie-Henriette DU BUIT }}{ }^{\text {b }}$, Jean-Jacques VAYNE ${ }^{\text {a }}$ \\ ${ }^{a}$ IFREMER, Station de La Rochelle, Place du Séminairc, BP 7, 17137 L'Houmcau, France \\ ${ }^{b}$ Laboratoire de Biologie marine du Muséum national d'Histoire naturelle et du Collège de France, 29182 Concarneau, \\ France
}

(Rcvised 21/11/97, acceptcd 14/01/98)

\begin{abstract}
The records of tropical fishes and the warming of the European Atlantic waters. A recapitulation of the records of tropical fishes from European Atlantic waters shows that $67.6 \%$ were fishes caught from the upper slope, between approximately 200 and $600 \mathrm{~m} ; 19.8 \%$ were fishes caught from the continental shelf; and $13.5 \%$ were specimens caught from the middle slope, between 700 and $1300 \mathrm{~m}$. Since 1963, the upper slope species have made regular northward range extensions off of south Portugal to north-western Ireland (about $55^{\circ} 30^{\prime} \mathrm{N}$ ), more and less rapidly, about 30 years for Cyttopsis roseus and only 6 years for Sphoeroides pachygaster. The continental shelf species, observed from 1969 but mostly from 1981, have a northward range to south-eastern Ireland (about $52^{\circ} \mathrm{N}$ ), but $65.2 \%$ of them have been caught off the south of the Bay of Biscay. The middle slope species, recorded only from 1991 according to the development of the deep fishery, were caught between $48^{\circ} \mathrm{N}$ and $60^{\circ} \mathrm{N}$. The northward range extension of upper slope species and the higher frequency of records of continental shelf species from the southern part of the Bay of Biscay coincide with the investigations on the warming of the south-north current in the upper slope of northern Spain and of the south French Atlantic continental shelf. (c) Elsevier, Paris
\end{abstract}

\section{biodiversity / tropical fishes / warming / European Atlantic}

Résumé - Un bilan des observations de poissons tropicaux en Atlantique nord-est montre que 66,7\% d'entre elles concernent des espèces vivant au niveau de la partie supérieure du talus, environ entre 200 et $600 \mathrm{~m} ; 19,8 \%$ sur le plateau continental et $13,5 \%$ sur le talus moyen entre 700 et $1300 \mathrm{~m}$. Les poissons tropicaux du talus supérieur, depuis 1963, ont progressé assez régulièrement vers le nord, du sud du Portugal jusqu'au nord-ouest de l'Irlande $\left(55^{\circ} 30^{\prime} \mathrm{N}\right)$, plus ou moins rapidement, en trente ans pour Cyttopsis roseus, en six ans pour Sphoeroides pachygaster. Les espèces tropicales du plateau continental, observées à partir de 1969 dans les eaux européennes mais surtout à partir de 1981, ont été signalées jusqu' au sud-est de l'Irlande $\left(52^{\circ} \mathrm{N}\right)$ mais $65,2 \%$ d'entre elles ont été capturées dans la partie sud du golfe de Gascogne. Les espèces plus profondes du talus moyen signalées seulement depuis 1991, avec le développement de l'exploitation intensive des grands fonds, ont été capturées entre $48^{\circ}$ et $60^{\circ} \mathrm{N}$. La progression vers le nord des espèces du talus supérieur et leur plus grande fréquence sur le plateau continental du sud du golfe de Gascogne coïncident avec la mise en évidence du réchauffement des eaux, respectivement du courant de pente sud-nord en Espagne et de la surface dans le golfe de Gascogne. (C) Elsevier, Paris

biodiversité / poisson tropical / réchauffement / Atlantique européen

* Correspondence and reprints 


\section{INTRODUCTION}

Au cours du $4^{e}$ Colloque d'Océanographie du golfe de Gascogne, Le Cann et Pingree avaient présenté une synthèse des travaux en océanographie physique effectués dans la région [2]. Ces auteurs ont montré un réchauffement général des eaux du courant sud-nord longeant la pente conlinentale au nord-ouest de l'Espagne pendant la période de 1969 à 1992, l'augmentation de la température ćtant de $1{ }^{\circ} \mathrm{C}$ par décennic, cntre 1972 et 1992 . Particulic̀rement intéressés par ces résultats, après avoir constaté à plusieurs reprises la progression vers le nord de l'aire de répartition d'un zéidé profond, Zenopsis conchifer [6, 4], les auteurs ont décidé, à l'occasion du $5^{\mathrm{e}}$ Colloque de faire un bilan des observations concernant la présence de poissons à affinités tropicales le long des côtes atlantiques européennes puis de les confronter aux données hydrologiques traitant du réchauffement des eaux du talus au nord de l'Espagne [2] et des eaux de surface du plateau continental du Sud-Gascogne, résultats présentés par Koutsikopoulos au colloque de La Rochelle [1].

\section{MATÉRIEL ET MÉTHODES}

Un inventaire des captures de poissons à affinités tropicales, inconnus au nord du Portugal avant 1950, par les bateaux de pêche ou des navires océanographiques portugais, espagnols, français, anglais et irlandais a été établi. Il comprend 111 observations dont 89 ont été signalées antérieurement, les 22 autres n'étant pas publiées. La longueur des articles du Colloque étant limitée, les données sur la distribution géographique et bathymétrique de chaque espèce, les indications sur les captures et lorsqu'elles existent, les références bibliographiques correspondantes ont été publiées séparément [5]. Le bilan des observations est présenté dans le tableau $I$.

\section{RÉSULTATS}

Les 121 exemplaires considérés ont été récoltés au cours de 111 pêches ; 84 appartiennent à des espèces vivant généralement au niveau du haut du talus entre 200 et $600 \mathrm{~m}$ environ : Zenopsis conchifer, Cyttopsis roseus, Chaunax spp, Sphoeroides pachygaster et Grammicolepis brachiusculus. Ensuite, 23 individus sont des poissons pélagiques, dont 16 appartiennent à des espèces côtières ou néritiques vivant au-dessus du plateau continental: Tarpon atlanticus, Seriola dumerili, Lichia amia, Pomatomus saltator, Aluterus monoceros et Seriola carpenteri; 7 à des espèces océaniques : Seriola rivoliana. Enfin 14 sont des poissons du talus moyen pêchés entre

Tableau I. Les observations, le long des côtes atlantiques européennes, de poissons à affinités tropicales, non signalés au nord du Portugal avant 1950, avec indications du nombre d'exemplaires, de leurs tailles extrêmes, et des profondeurs de capture extrêmes et moyennes (d'après Quéro et al., 1997).

Table I. The records, along the European Atlantic coasts, of fishes originated from tropical waters, non-recorded from north Portugal before 1950, with data on the number of specimens and their extreme sizes, and the extreme and mean depths of catches. (From Quéro et al. [5]).

\begin{tabular}{|c|c|c|c|c|c|}
\hline \multirow{2}{*}{ Espèces } & \multicolumn{2}{|c|}{ Nombre } & \multirow{2}{*}{$\begin{array}{c}\text { Tailles } \\
\text { (cm) }\end{array}$} & \multicolumn{2}{|c|}{ Profondeurs observées (m) } \\
\hline & observations & exemplaires & & extrêmes & moyennes \\
\hline Zenopsis conchifer & 34 & 36 & $17,2-67$ & $100-500$ & 280 \\
\hline Cyttopsis roseus & 13 & 19 & $10,5-24,5$ & $150-500$ & 320 \\
\hline Chaunax spp. & 15 & 16 & $13,8-29$ & $165-1300$ & 600 \\
\hline Sphoeroides pachygasler & 12 & 12 & $12,5-37$ & $120-270$ & 185 \\
\hline Tarpon atlanticus & 7 & 7 & $135-193$ & $7-80$ & \\
\hline Seriola rivoliana & 6 & 7 & $39-40$ & $30-40$ & 35 \\
\hline Hoplostethus cadenati & 6 & 6 & $19,5-26$ & $950-1300$ & 1050 \\
\hline Allocyttus verrucosus & 3 & 3 & 37 & & \\
\hline Dibranchus atlanticus & 3 & 3 & $13-16$ & & \\
\hline Seriola dumerili & 3 & 3 & & & \\
\hline Diretmoides parini & 2 & 2 & $26-35$ & 900 & \\
\hline Lichia amia & 2 & 3 & & & \\
\hline Pomatomus saltator & 2 & 2 & & & \\
\hline Aluterus monoceros & 1 & 1 & & & \\
\hline Grammicolepis brachiusculus & 1 & 1 & 53 & 600 & \\
\hline Seriola carpenteri & 1 & 1 & 55 & 82 & \\
\hline
\end{tabular}




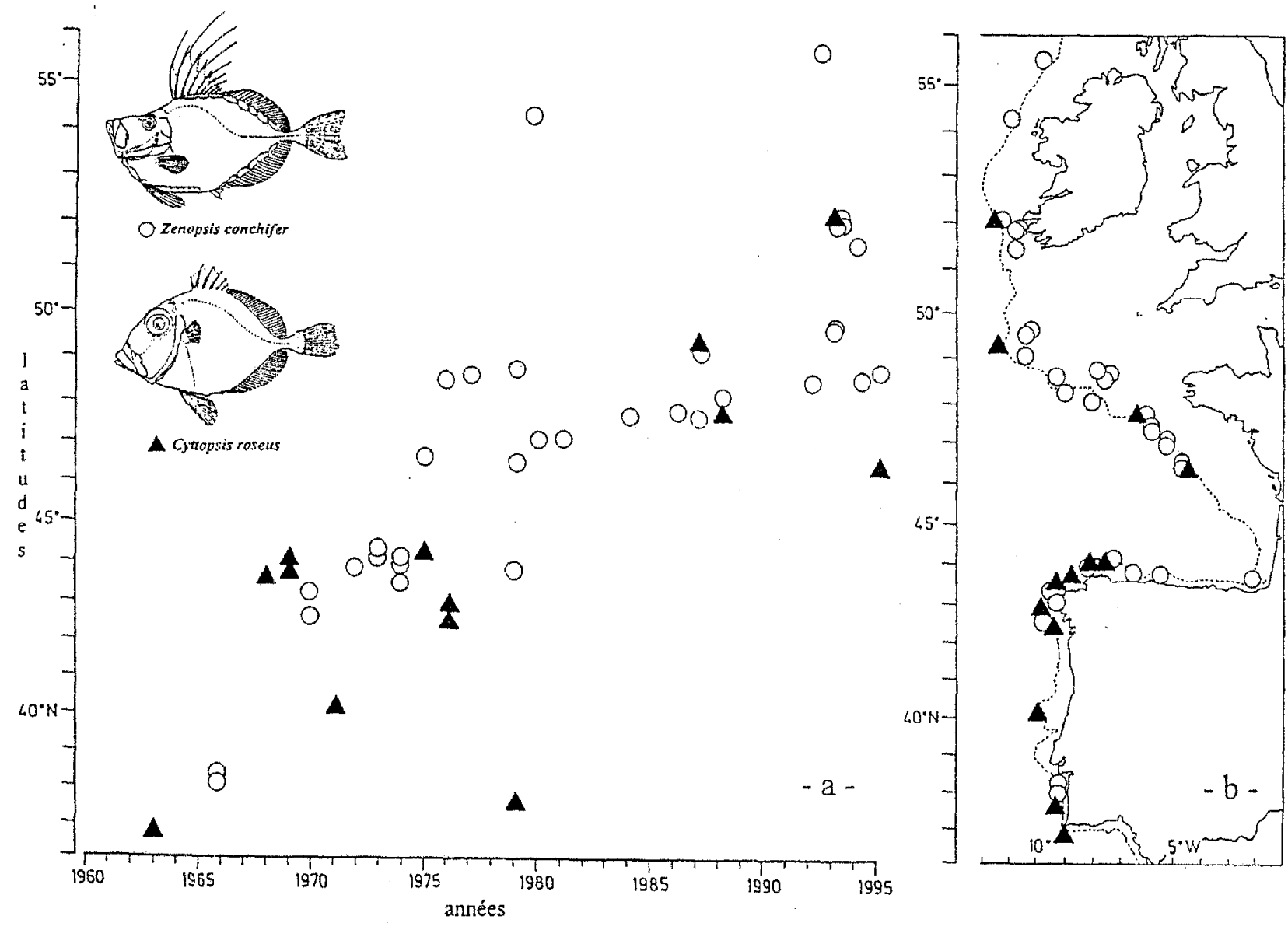

Figure 1. (a) Distribution selon les latitudes et les années, de 1960 à 1995 et (b) localisation géographique des captures de Zenopsis conchifer et Cyttopsis roseus le long des côtes atlantiques européennes.

Figure 1. (a) Distribution according to latitudes and years from 1960 to 1995 and (b) geographical distribution of catches of Zenopsis conchifer and Cyttopsis roseus along the European Atlantic coasts.

environ 900 et $1300 \mathrm{~m}$ : Hoplostethus cadenati, Allocytus verrucosus, Dibranchus atlanticus et Diretmoides parini.

Les cinq espèces du talus supérieur totalisent les deux tiers des observations $(67,6 \%$ de l'ensemble) et un peu plus des deux tiers des spécimens $(69,4 \%$ du nombre total).

Zenopsis conchifer (Lowe, 1852), poisson de grande taille généralement rapporté par les pêcheurs, est abondant au sud de $21^{\circ} \mathrm{N}$ entre 200 et $400 \mathrm{~m}$ de profondeur. II a été observé le long des côtes européennes à 34 reprises (36 exemplaires). Signalé pour la première fois en Europe dans les eaux portugaises en 1966 (figure 1), il progresse lentement vers le nord, atteignant en quatre ans (1970) le nord-ouest de l'Espagne, en neuf ans (1975) le golfe de
Gascogne, en 14 ans (1980) l'ouest de l'Irlande et en 27 ans (1993) le nord-ouest de l'Irlande.

Cyttopsis roseus (Lowe, 1843), poisson plus petit (tableau $I$ ) est abondant sur les côtes d'Afrique vers $26^{\circ} \mathrm{N}$ entre 400 et $600 \mathrm{~m}$ de profondeur. Il a été observé au large des côtes européennes à 13 reprises (19 exemplaires recensés). Signalé une fois au début du siècle au sud du Portugal, il a été capturé de nouveau en 1963 (figure 1). Sa progression vers le nord est voisine de celle de Zenopsis conchifer, atteignant en cinq ans (1968) le nord-ouest de l'Espagne, en 24 ans (1987) le plateau Cellique, en 30 ans (1993) le sud-ouest de l'Irlande.

Le genre Chaunax est représenté dans les eaux atlantiques européennes par deux espèces, Chaunax pictus, 

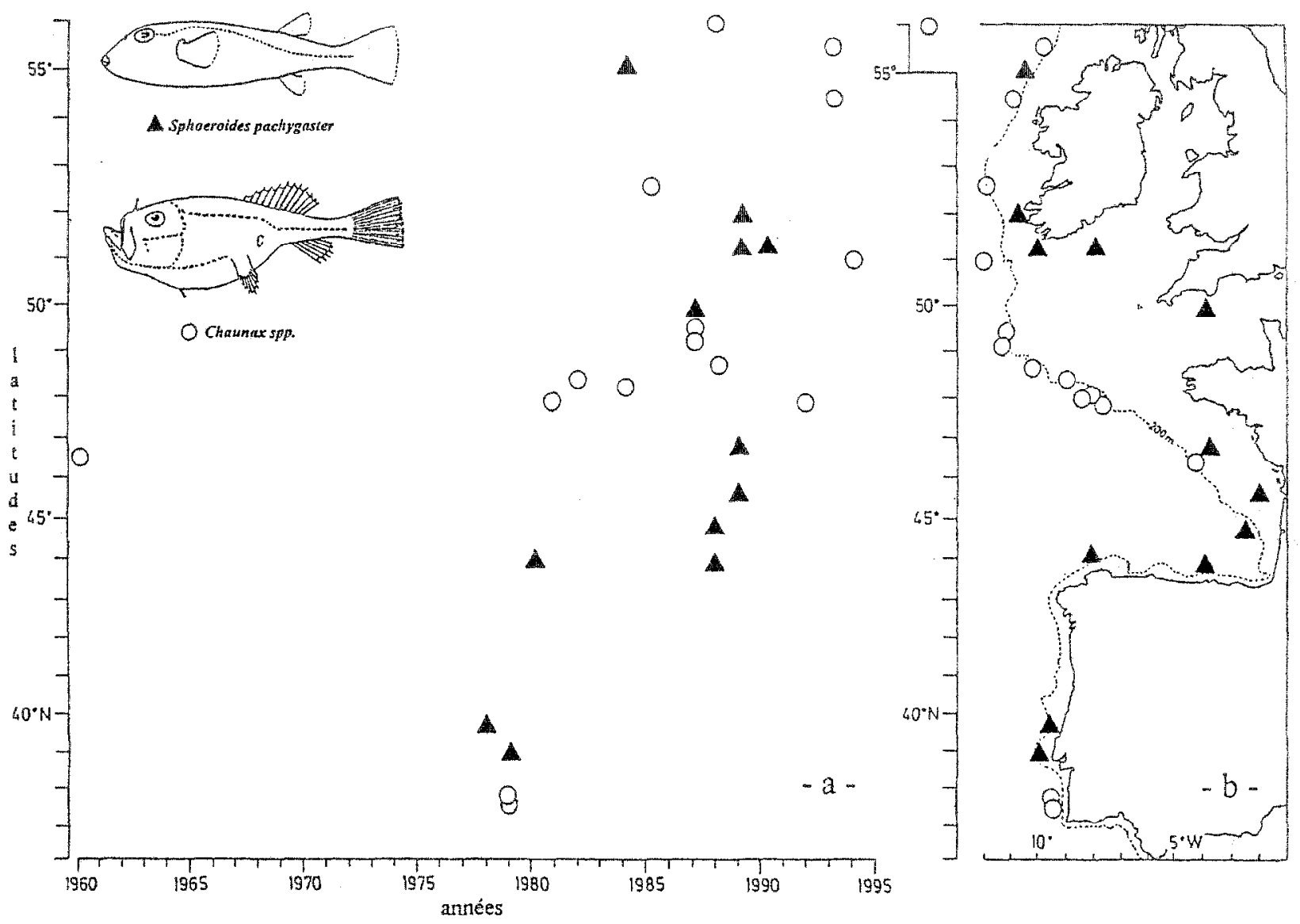

Figure 2. (a) Distribution selon les latitudes et les années, de 1960 à 1995 et (b) localisation géographique des captures de Sphoeroirios pachygaster, Chaunax spp et Grammicolepis brachiusculus le long des côtes atlantiques européennes.

Figure 2. (a) Distribution according to latitudes and years from 1960 to 1995 and (b) geographical distribution of catches of Sphoeroides pachygaster, Chaunax spp. and Grammicolepis brachiusculus along the European Atlantic coasts.

Lowe, 1846, et Chaunax suttkusi, Caruso, 1989. La détermination des exemplaires antérieurs à 1989 , date de la révision de Caruso, non mis en collection, est incertaine. Benthopélagiques, ces poissons habitent le plus souvent entre 275 et $625 \mathrm{~m}$ de profondeur. Cités à deux reprises au Portugal au début du siècle, ils ont été pêchés dans le golfe de Gascogne en 1960 (figure 2). Toutefois, c'est seulement à partir de 1979 au Portugal que les captures deviennent moins exceptionnelles avec 14 observations (15 exemplaires). Le genre Chaunax est signalé dans le golfe de Gascogne en 1981, quatre ans après (1985) à l'ouest de l'Irlande et douze ans plus tard (1993) au nord ouest de l'Irlande.

Sphoeroides pachygaster (Müller \& Troschel, 1848) vit dans les mers chaudes généralement entre 100 et $400 \mathrm{~m}$ de profondeur. Cité une fois au Portugal en 1931, il sera capturé douze fois dans les eaux européennes de 1978 à 1990. Sa progression vers le nord est très rapide, atteignant en deux ans le nord-ouest de l'Espagne (1980) et en six ans le nord-ouest de l'Irlande (1984).

Grammicolepis brachiusculus, Poey, 1873, poisson benthopélagique des mers tropicales et subtropicales entre 400 et $800 \mathrm{~m}$ de profondeur, n'a été capturé qu'une seule fois en 1966 au nord-ouest de l'Espagne.

Les espèces côtières ou néritiques d'affinités tropicales ont été signalées 22 fois (soit 19,8\% des observations) et sont représentées par 23 spécimens (19\% de la totalité des poissons). Ce nombre d'individus répertoriés est donc nettement inférieur aux résultats obtenus sur le haut du talus continental mais la diversité spécifique (sept espèces) est plus élevée. La progression vers le nord de ces 

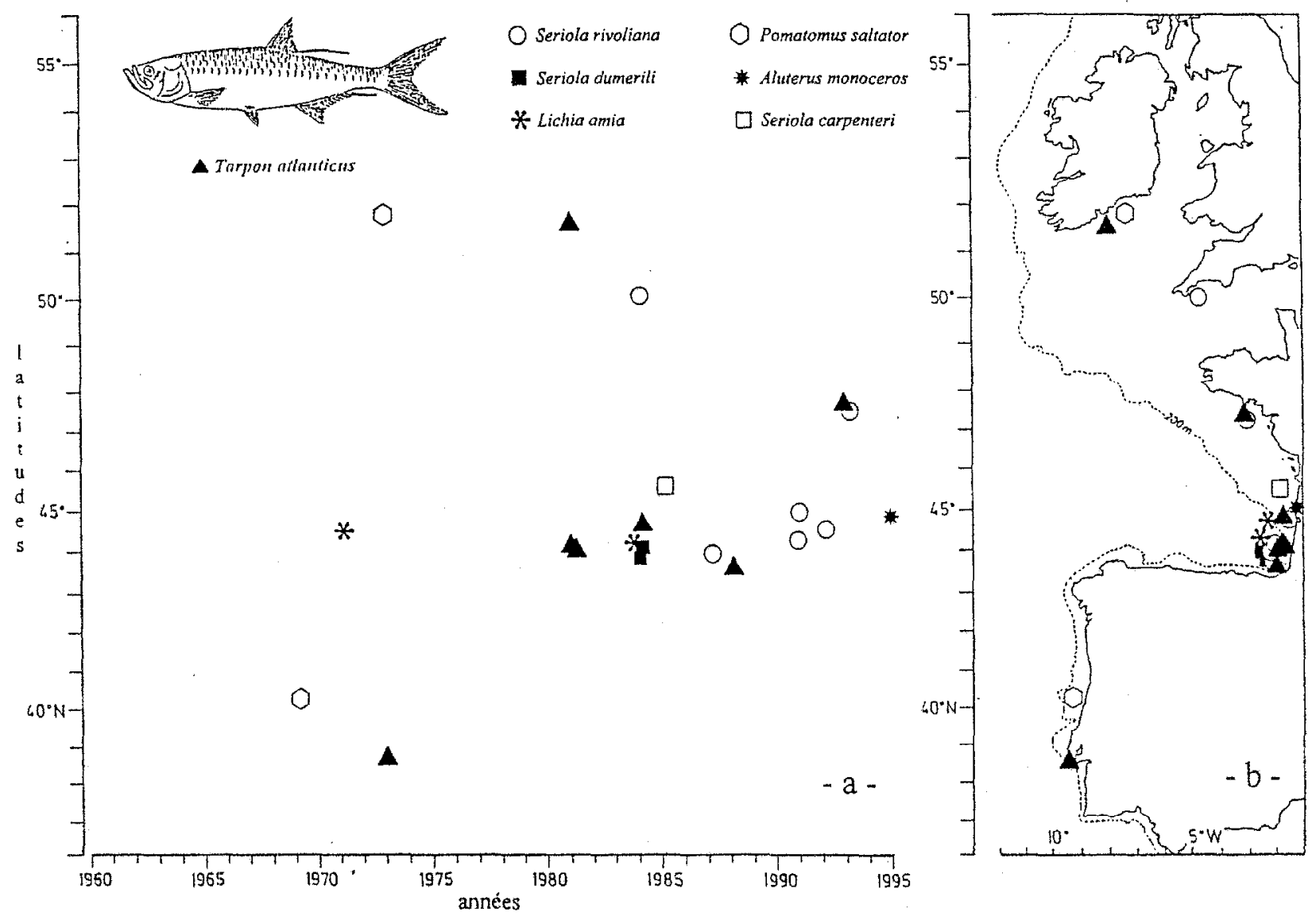

Figure 3. (a) Distribution selon les latitudes et les années de 1960 à 1995 et (b) localisation géographique des captures de Tarpon atlanticus, Seriola rivoliana, Seriola dumerili, Lichia amia, Pomatomus saltator, Aluterus monoceros et Seriola carpenteri le long des côtes atlantiques européennes.

Figure 3. (a) Distribution according to latitudes and years from 1960 to 1995 and (b) geographical distribution of catches of Tarpon atlanticus, Seriola rivoliana, Seriola dumerili, Lichia amia, Pomatomus saltator, Aluterus monoceros and Seriola carpenteri along the European Atlantic coasts.

espèces est également moins régulière (figure 3) ; après une première indication en 1951 (non figuré) d'une Seriola dumerili au sud-ouest de la Grande-Bretagne, nous avons répertorié, de 1969 à 1973, quatre observations géographiquement dispersées entre le Portugal ( 2 ex.), le golfe de Gascogne (1 ex.), le sud-ouest de l'Irlande (1 ex.) ; les poissons signalés ont été plus fréquents à partir de 1981 ; après 1984 , ils concernent uniquement le golfe de Gascogne et plus particulièrement la partie méridionale. Ces poissons tropicaux néritiques ont été le plus souvent capturés dans des zones peu profondes, principalement entre 30 et $80 \mathrm{~m} ; 73,9 \%$ d'entre eux proviennent du golfe de Gascogne et plus de la moilié $(65,2 \%)$ de la partie sud.
Les quatre espèces tropicales pêchées sur le talus moyen, entre 700 et $1300 \mathrm{~m}$ de profondeur, constituent le groupe le moins bien représenté, seulement $12,6 \%$ des observations et 11,6\% des. spécimens. La figure 4 met en évidence l'absence de signalement antérieur à 1991 et une répartition des observations entre $48^{\circ} \mathrm{N}$ et $60^{\circ} \mathrm{N}$ avec un maximum d'abondance au nord-ouest de l'Irlande. L'exploitation commerciale du talus continental jusqu'à $1300 \mathrm{~m}$ a commencé vers 1989 et s'est généralisée en 1991 au nord du golfe de Gascogne, surtout au large des Iles Britanniques.

L'espèce la mieux représentée Hoplostethus cadenati, Quéro, 1974, n'était connue antérieurement qu'au sud 


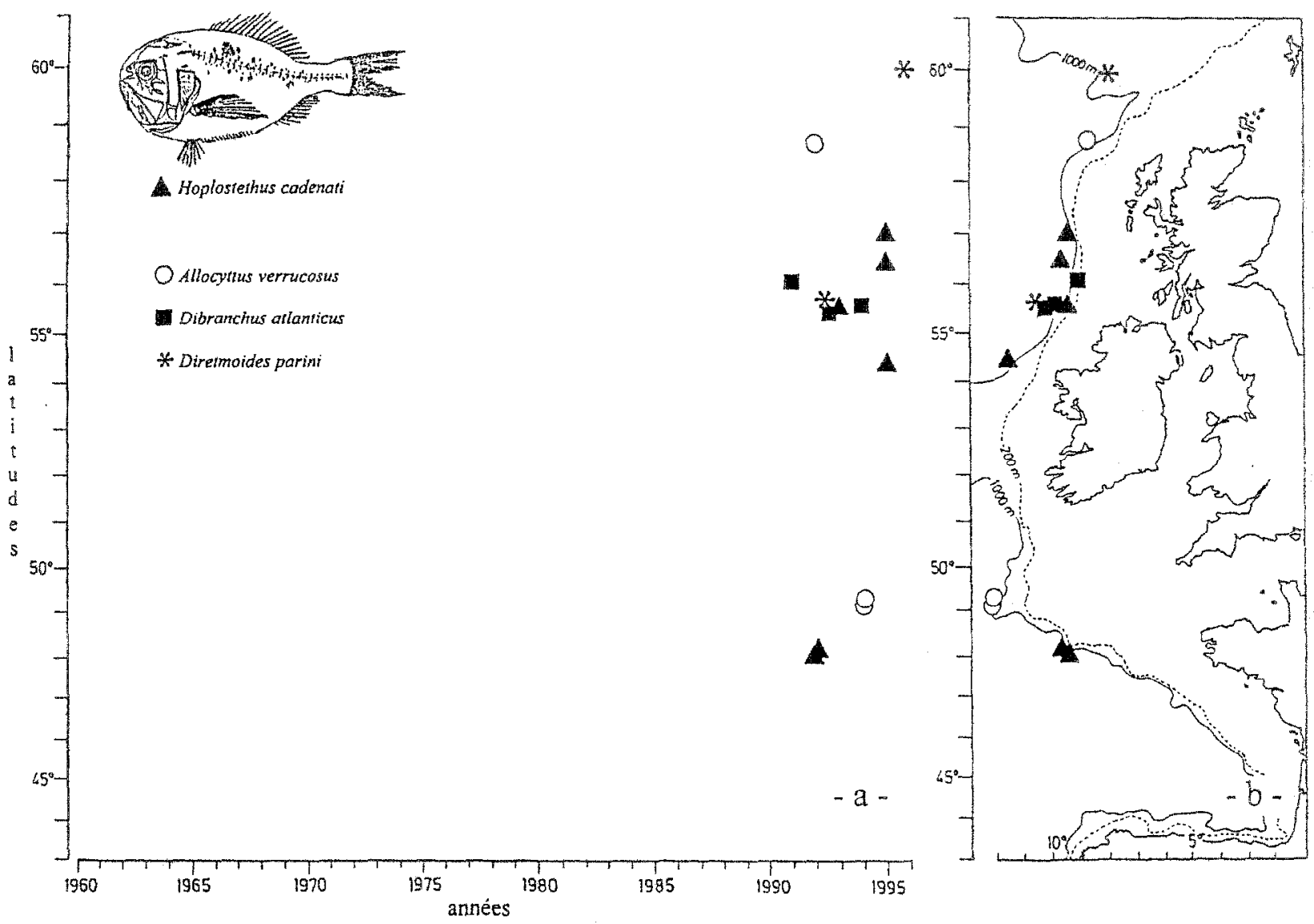

Figure 4. (a) Distribution selon les latitudes et les années de 1960 à 1995 et (b) localisation géographique des captures d’Hoplostethus cadenati, Allocyttus verrucosus, Dibranchus atlanticus et Diretmoides parini le long des côtes atlantiques européennes.

Figure 4. (a) Distribution according to latitudes and years from 1960 to 1995 and (b) geographical distribution of catches of Hoplostethus cadenati, Allocyttus verrucosus, Dibranchus atlanticus and Diretmoides parini along the European Atlantic coasts.

des Iles Canaries $\left(27^{\circ} 30 \mathrm{~N}\right)$. Elle n'était fréquente qu'au sud du cap Corveiro $\left(21^{\circ} 50^{\circ} \mathrm{N}\right)$ entre 200 et $1000 \mathrm{~m}$ de profondeur. Depuis 1982, six spécimens ont été répertoriés le long du talus continental du plateau Celtique et jusqu'à l'ouest de $1^{\prime} E \operatorname{cosse}\left(57^{\circ} \mathrm{N}\right)$.

\section{DISCUSSION}

Nous constatons une double coincidence : la première entre la mise en évidence sur la côte nord de l'Espagne entre 1972 et 1992 d'un réchauffement des eaux atlantiques du courant de pente vers le nord, d'environ $2^{\circ} \mathrm{C}$ [2] et la progression de poissons tropicaux le long de la partie supérieure du talus où circule ce courant (figures 1,2); la deuxième entre la démonstration d'une hausse de la tem- pérature moyenne hivernale et estivale plus forte qu'ailleurs dans le sud du golfe de Gascogne, $1,4^{\circ} \mathrm{C}$ entre 1972 et 1993 [1] et la concentration d'environ deux tiers des captures d'especes tropicales côtières dans cette partie du golfe de Gascogne (figure 3).

Nous constatons également que les espèces qui présentent l'extension vers le nord la plus régulière (figures 1,2) sont localisées sur la partie supérieure du talus où circule le courant sud-nord.

Les captures les plus septentrionales concernent les espèces les plus profondes : $60^{\circ} \mathrm{N}$ pour celles vivant entre 700 et $1300 \mathrm{~m} ; 55^{\circ} 30^{\prime} \mathrm{N}$ pour celles vivant entre 200 et $600 \mathrm{~m} ; 52^{\circ} \mathrm{N}$ pour les poissons du plateau continental.

Dans une note sur la capture de S. pachygaster au nordouest de l'Irlande, Wheeler \& van Oijen [7] émettent 
l'hypothèse que les poissons d'affinités tropicales signalés dans les eaux européennes pourraient venir de l'Atlantique ouest, entraînés par la dérive Nord-Atlantique. Ce n'est pas le cas de H. cadenati et $C$. roseus; le premier est uniquement africain et le second, commun au sud du Maroc, n'a été signalé que récemment dans l'Atlantique Centre-Ouest.

\section{CONCLUSION}

La biodiversité ichtyologique des eaux atlantiques européennes, si elle s'appauvrit du fait de la pêche comme nous l'avons montré avec l'exemple de la disparition d'un requin, Echinorhinus brucus et la forte raréfaction des grands poissons cartilagineux vivant sur le fond [3], s'enrichit d'espèces tropicales du fait de modifications climatiques. Ces espèces nouvelles pour notre région y restent toutefois marginales; ce n'est pas toujours le cas. Balistes carolinensis par exemple, dont nous n'avons pas tenu compte ici puisque signalé en Europe avant 1950, ne répondait pas à nos critères de sélection; il était autrefois rare en Europe. Il est devenu si abondant qu'il figure maintenant dans les statistiques de pêche. Nous constatons également l'extension vers le nord d'autres espèces de sparidés et soléidés d'affinités tropicales. Ce phénomène mériterait plus d'attention.

\section{RÉFÉRENCES}

[1] Koutsikopoulos C., Beillois P., Leroy C., Taillefer F., Temporal trends and spatial structures of the Sea Surface Temperature in the Bay of Biscay, $V^{e}$ Colloque international du golfe de Gascogne, La Rochelle (1996).

[2] Le Cann B., Pingree R., Circulation dans le golfe de Gascogne : une revue de travaux récents, Actas IV Coloqu. internac. Oceanogr. Golfo Vizcaya/TV Coll. internat. golfe Gascogne, (1995) 217-234.

[3] Quéro J.C., Cendrero O., Incidence de la pêche sur la biodiversité ichtyologique marine : le bassin d'Arcachon et le plateau continental Sud-Gascogne, Cybium 20 (4) (1996) 323-356.
[4] Quéro J.C., Décamps P., Duron, M., Fonteneau J., Observations ichtyologiques effectuées en 1981, Ann. Soc. Sci. Nat. Charente-Marit. 6 (8) (1981) 837-845.

[5] Quéro J.C., Du Buit M.H., Vayne J.J., Les captures de poissons à affinités tropicales le long des côtes atlantiques européennes. Ann. Soc. Sci. Nat. Charente-Marit. 8 (6) (1997) 651-73.

[6] Quéro J.C., Verron R., Cattin Y., Observations ichtyologiques effectuées au port de La Rochelle en 1976, Ann. Soc. Sci. Nat. Charente-Marit. 6 (1) (1977) 230- 241.

[7] Wheeler A., van Oijen M.J.P., The occurrence of Sphoeroides pachygaster (Osteichthyes-Tetrandontiformes) off North-West Ireland, Zool. Meded. Leiden 59 (1985) 101-107. 